University of Nebraska - Lincoln

DigitalCommons@University of Nebraska - Lincoln

2014

\title{
A probabilistic method for constructing wave time-series at inshore locations using model scenarios
}

J. W. Long

USGS Coastal and Marine Geology Program, jwlong@usgs.gov

N. G. Plant

USGS Coastal and Marine Geology Program

P. S. Dalyander

USGS Coastal and Marine Geology Program

D. M. Thompson

USGS Coastal and Marine Geology Program

Follow this and additional works at: https://digitalcommons.unl.edu/usgsstaffpub

Long, J. W.; Plant, N. G.; Dalyander, P. S.; and Thompson, D. M., "A probabilistic method for constructing wave time-series at inshore locations using model scenarios" (2014). USGS Staff -- Published Research. 834.

https://digitalcommons.unl.edu/usgsstaffpub/834

This Article is brought to you for free and open access by the US Geological Survey at DigitalCommons@University of Nebraska - Lincoln. It has been accepted for inclusion in USGS Staff -- Published Research by an authorized administrator of DigitalCommons@University of Nebraska - Lincoln. 


\title{
A probabilistic method for constructing wave time-series at inshore locations using model scenarios
}

\author{
J.W. Long *, N.G. Plant, P.S. Dalyander, D.M. Thompson \\ USGS Coastal and Marine Geology Program, St. Petersburg Coastal and Marine Science Center, St. Petersburg, FL, USA
}

\section{A R T I C L E I N F O}

\section{Article history:}

Received 18 September 2013

Received in revised form 25 March 2014

Accepted 26 March 2014

Available online 4 May 2014

\section{Keywords:}

Coastal modeling

Waves

Wave prediction

Probabilistic models

Wave transformation

\begin{abstract}
A B S T R A C T
Continuous time-series of wave characteristics (height, period, and direction) are constructed using a base set of model scenarios and simple probabilistic methods. This approach utilizes an archive of computationally intensive, highly spatially resolved numerical wave model output to develop time-series of historical or future wave conditions without performing additional, continuous numerical simulations. The archive of model output contains wave simulations from a set of model scenarios derived from an offshore wave climatology. Timeseries of wave height, period, direction, and associated uncertainties are constructed at locations included in the numerical model domain. The confidence limits are derived using statistical variability of oceanographic parameters contained in the wave model scenarios. The method was applied to a region in the northern Gulf of Mexico and assessed using wave observations at $12 \mathrm{~m}$ and $30 \mathrm{~m}$ water depths. Prediction skill for significant wave height is 0.58 and 0.67 at the $12 \mathrm{~m}$ and $30 \mathrm{~m}$ locations, respectively, with similar performance for wave period and direction. The skill of this simplified, probabilistic time-series construction method is comparable to existing large-scale, high-fidelity operational wave models but provides higher spatial resolution output at low computational expense. The constructed time-series can be developed to support a variety of applications including climate studies and other situations where a comprehensive survey of wave impacts on the coastal area is of interest.
\end{abstract}

Published by Elsevier B.V.

\section{Introduction}

There is an increasing need for accurate, site-specific, and timely estimates of coastal wave properties that can be used to design marine infrastructure, perform coastal vulnerability assessments (e.g., Stockdon et al., 2012), evaluate potential sites for wave energy extraction (Defne et al., 2009; Reikard, 2009), and drive empirical (Stockdon et al., 2006; Yates et al., 2009) and process-based (e.g., XBeach, Roelvink et al., 2009) nearshore models. Wave properties for these applications are generally obtained from sparse buoy arrays (hindcast or nowcast) or a variety of process-based wave transformation models (hindcast, nowcast, and forecast). The models can range significantly in resolution, scale, and computational expense. Oftentimes highly spatially-resolved nearshore models are run deterministically by initializing with waves observed at buoy locations and allowing the model to transform the waves to the site of interest. However, these can be computationally expensive depending on the length of the time-series and number of wave conditions required, especially if statistical uncertainties are required (e.g., sensitivity testing). Alternately, operational wave forecasts, forced with predicted global and basin-scale wind fields, archive forecast

\footnotetext{
* Corresponding author.

E-mail address: jwlong@usgs.gov (J.W. Long).
}

output for future use in hindcast studies (e.g., WAVEWATCH-III@, Tolman (2008)). Despite the overall good skill of these forecasts, the resolution is typically $\mathrm{O}(3-7 \mathrm{~km})$ which is insufficient to resolve some important shelf-scale features and nearshore wave transformation processes.

In order to improve the efficiency of wave prediction over operational or deterministic models, methods have been developed that exploit machine-learning techniques (e.g., Neural Networks, fuzzy logic, and Bayesian methods) to estimate wave characteristics at one location given information at another (e.g., Camus et al., 2011; Londhe, 2008; Londhe and Panchang, 2007; Plant and Holland, 2011a,b). While this is useful, for example, to fill data gaps at observational buoys, these techniques ("machine learning") do not provide information on the physical transformation of waves between the sites and require historical data at both locations to train the algorithms and determine the relationship between the wave fields at each site. The applicability of these methods is limited when information is desired at locations where no buoy has ever been deployed. One solution if observational data at the target site are unavailable is to use deterministic model runs to train the machine-learning models; however, the efficiency benefit of the machine-learning technique is then lost.

In a similar fashion, O'Reilly and Guza (1993) compared simple refraction and refraction-diffraction models and, from the model output, derived wave energy transformation coefficients to estimate coastal 
wave properties based on offshore wave information. The coefficients are a function of frequency and direction and are region-specific. The method is computationally efficient but is only applicable for lowfrequency swell in regions where offshore wave conditions are spatially homogeneous. Similar to the operational and deterministic methods, statistical confidence limits are not reported.

Here we present a probabilistic time-series construction technique that uses a process-based coastal and nearshore numerical wave model to transform deep water waves inshore. In contrast to the wave transformation or machine learning methods, this probabilistic method can construct a continuous wave time-series over a spatial domain of interest using a set of climatologically-based numerical simulations. The numerical model accounts for physical wave transformation processes, does not require algorithm training and does not require computationally expensive model runs. In addition, it is not limited to sites with available observations and may be applied to analyze possible future scenarios. We demonstrate the validity of the technique using time-series that span multiple years and locations and derive statistical uncertainty estimates based on historical distributions of the wave climate.

The probabilistic time-series construction method is described in Section 2. Results from two hindcasted probabilistic time-series constructions at locations in $12 \mathrm{~m}$ and $30 \mathrm{~m}$ water depths are presented in Section 3. In Section 4 we discuss limitations and sensitivity of the technique to some of our assumptions, and conclusions are synthesized in Section 5.

\section{Methods}

\subsection{Derivation of wave scenarios}

The probabilistic method relies on the establishment of a discrete set of climatologically-derived base model simulations, or wave model scenarios, representing the wave conditions within the domain under a variety of offshore conditions. Wave model scenarios were defined from a climatological binning of offshore wave observations. The data used for the climatological assessment were obtained from the National Data Buoy Center (NDBC) buoy 42040 from April 2010 to May 2012. This buoy is located in the Gulf of Mexico in approximately $165 \mathrm{~m}$ water depth (labeled 42040 in Fig. 1). The wave observations from this time period, which peaked at $5.1 \mathrm{~m}$, were divided into 5 significant wave height $\left(H_{s}\right)$ bins corresponding to $0 \mathrm{~m}<H_{s} \leq 0.5 \mathrm{~m}, 0.5 \mathrm{~m}<$ $H_{s} \leq 1.0 \mathrm{~m}, 1.0 \mathrm{~m}<H_{s} \leq 1.5 \mathrm{~m}, 1.5 \mathrm{~m}<H_{s} \leq 2.0 \mathrm{~m}$, and $H_{s}>2.0 \mathrm{~m}$ and 16 wave direction bins, each spanning $22.5^{\circ}$, from 0 to $360^{\circ}$ (Fig. 2). The average of all the observed wave heights and directions that fall within each of the 80 climatological bins defines the targeted climatological conditions, and thus the offshore conditions for each wave model scenario.

To avoid the restriction of assuming homogeneity along the boundaries of our coastal model domain by applying only the targeted (average) significant wave height, peak period, and peak direction for each climatological bin, we used operational wave model forecast output along the boundaries of the domain. We performed a multivariate analysis to identify a best-match time in the buoy time-series when observed conditions most closely matched the average conditions for each climatological bin. The selected hour was required to come from a time period when the observed conditions met the targeted values for at least $6 \mathrm{~h}$, rather than from a time period when conditions were rapidly transitioning from one sea state to another. Despite not being included as a constraint, the wave period and offshore winds for the selected hour representing each bin were also found to closely match bin-averaged values.

For each of the 80 model scenarios, spatially-varying bulk wave characteristics (height, period, direction) from the best-match times were extracted from archived NOAA WAVEWATCH-III® $4(7.5 \mathrm{~km})$ U.S. East Coast and Gulf of Mexico wave model results (Tolman, 2008) along all boundaries of our coastal domain (Fig. 1). This method described the spatially heterogeneous wave climate over a large region

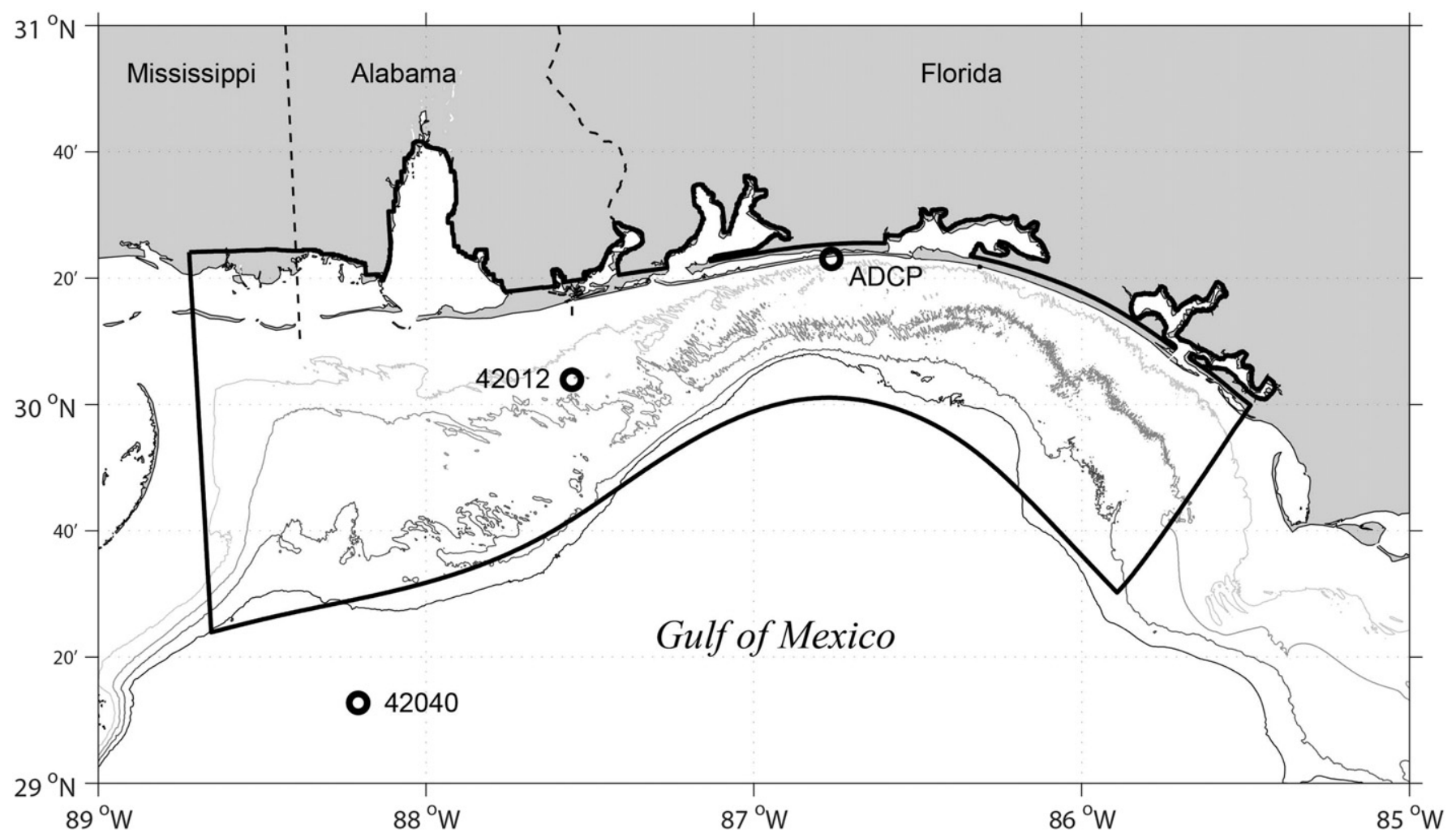

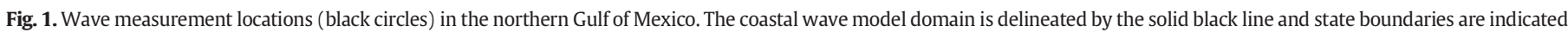
by the dashed lines. 


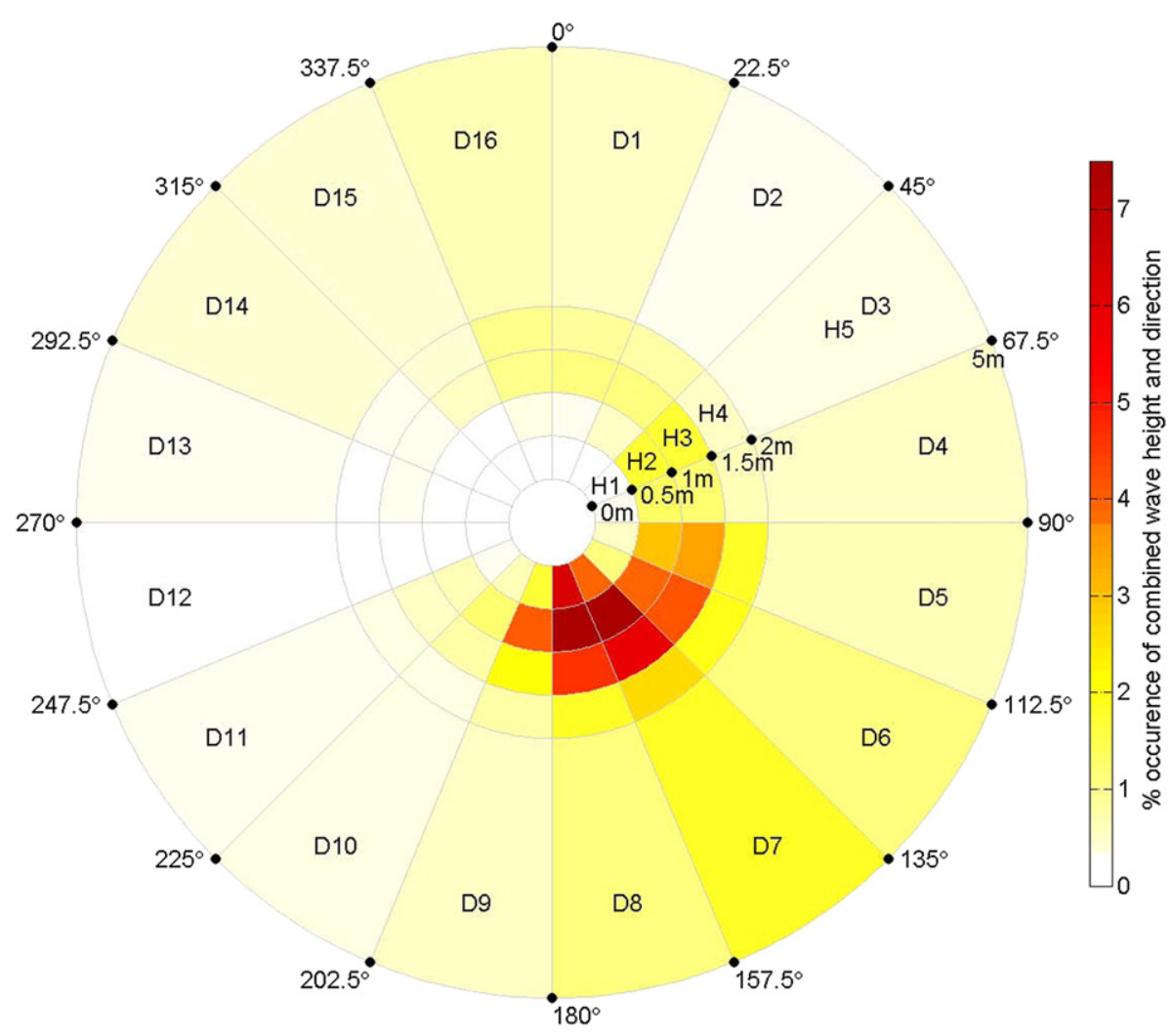

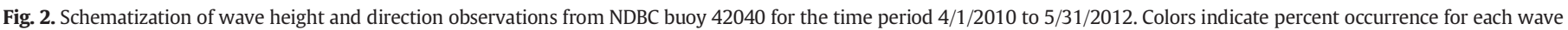

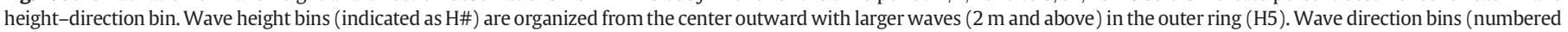
D1-D16) each span $22.5^{\circ}$, from 0 to 360 , radiating around diagram circumference.

rather than prescribing information only derived from a single buoy location. Buoy observations were used to populate the wave bins and identify the best-match time to limit the potential for numerical model error to propagate into the identification of the regional wave climatology. We confirmed that WAVEWATCH-III ${ }^{\circ}$ predictions at the offshore buoy closely matched the observed wave conditions for each of the 80 times chosen to initialize the scenarios.

This methodology results in 80 individual, discontinuous hours during the 26 month period that were used to represent the 80 bins defined by the climatological assessment. Each representative hour was then numerically simulated to transform the offshore waves characterized by the bin into the model domain. The resultant wave model scenario captures the spatially variant wave field at the hour in time chosen for each bin and is later used to represent the wave field for any other time when offshore wave conditions are matched to that climatological bin.

\subsection{Coastal model description}

The Simulating WAves Nearshore (SWAN) model (version 40.85; Booij et al., 1999) was used to transform the WAVEWATCH-III ${ }^{\circledR}$ wave information from the model boundaries to the inner shelf and nearshore regions of the northern Gulf of Mexico. The domain extends from Horn Island, MS to Crooked Island, FL with alongshore resolution of approximately $250 \mathrm{~m}$ and a cross-shore resolution varying from $2.5 \mathrm{~m}$ in the surf zone to $150 \mathrm{~m}$ offshore. In this implementation we include the effects of wind wave growth, spectral refraction, and wave shoaling as well as sinks of wave action including depth-induced breaking (Battjes and Janssen (1978) parameterization with default values of 1 and 0.73 for $\alpha$ and $\gamma$, respectively), whitecapping (Komen et al., 1984), and bottom friction (Hasselmann et al., 1973).
For each of the model scenarios, bulk wave characteristics extracted from the corresponding WAVEWATCH-III® output times are prescribed along the offshore and lateral boundaries of the coastal model domain with an assumed JONSWAP (JOint NOrth Sea WAve Project) spectral shape (Hasselmann et al., 1973). The boundary conditions were prescribed at every 30th grid cell, approximately matching the coarse resolution of the WAVEWATCH-III ${ }^{\circledR}$ product. Because we ran individual hours only, wave conditions and water levels ( $0 \mathrm{~m}$ above NAVD88) for these model scenarios do not change with time and SWAN was run in stationary mode. The wave models' directional domain covered a full circle with a resolution of $5^{\circ}$ ( 72 bins in total) and the frequency domain ranged from 0.05 to $1 \mathrm{~Hz}$ with logarithmic spacing. The spectral frequency and directional spreading parameters (default model parameters) were 3.3 and 2 (assuming $\cos ^{m}(\theta)$ distribution), respectively.

Forecasted wind speed and direction used as forcing for WAVEWATCH-III ${ }^{\circledR}$ were also obtained from the NOAA archives for each of the individual 80 model scenarios. These coarsely-resolved wind velocities were interpolated to our coastal model domain for each scenario to capture local wave generation. Similar to the wave boundaries, wind velocities were held stationary.

The model was used in stationary mode to generate steady-state wave conditions over the domain to represent the wave conditions for each climatological bin. Winds and spatially-variable offshore wave conditions at an hour when waves at the offshore buoy most closely matched the targeted average conditions for each bin were used to initialize each scenario. Hence, the coastal model provided 80 scenarios as a database of conditions that can be used to construct a time-series of wave conditions over anytime period of interest. Fig. 3 is an example of the predicted wave height for the scenario with $H_{s}>2 \mathrm{~m}$ and $135<\theta_{m}>157.5^{\circ}$. A map showing the standard deviation at each model grid cell is also given to show the variability in wave height at each location over the 80 scenarios. 

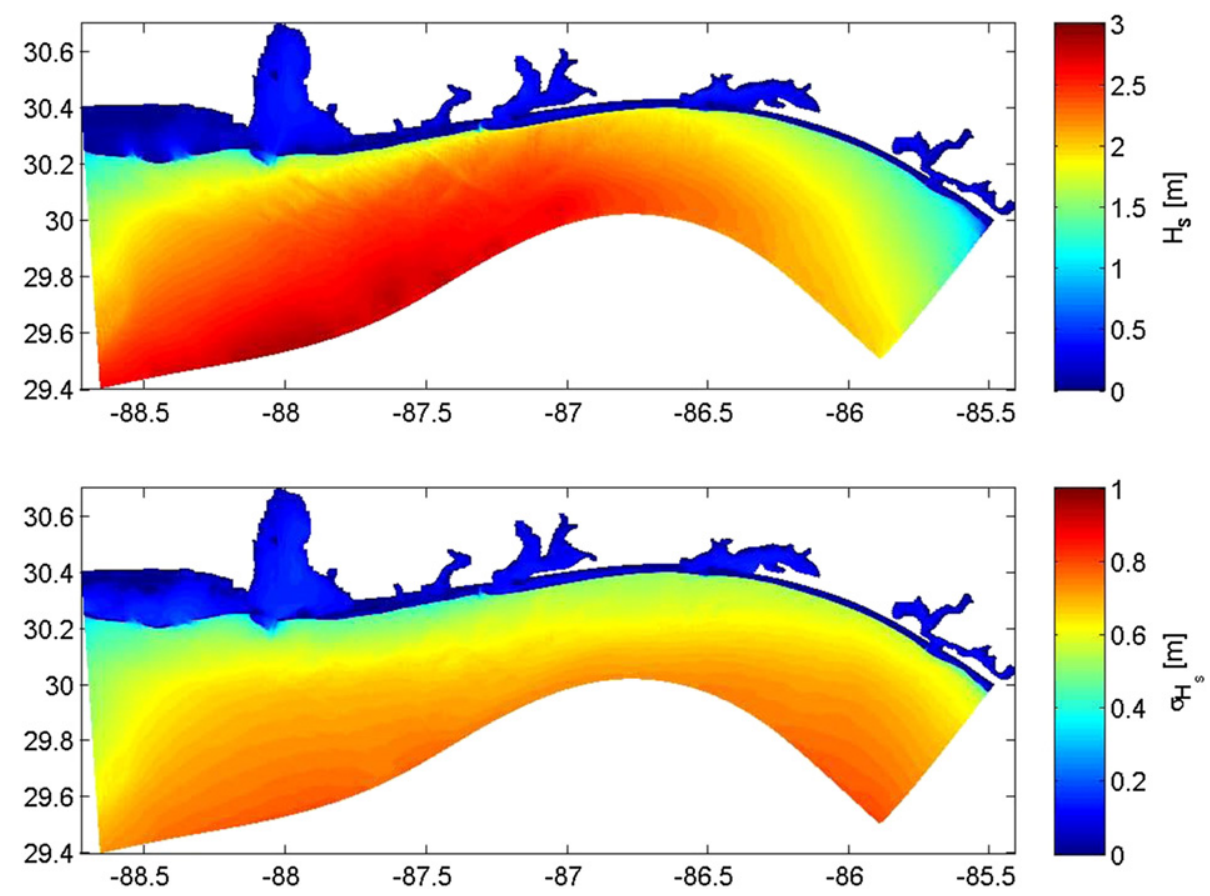

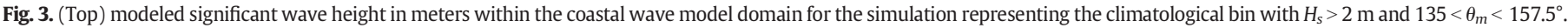
(Bottom) standard deviation of the modeled wave height in meters from all 80 climatological scenario simulations.

\subsection{Probabilistic time-series construction method}

Our goal was to skillfully construct time-series of wave height, direction, and period for any location in our northern Gulf of Mexico region of interest. To do this, we invoked a probability analysis based on the following: 1) offshore buoy observations, 2) output from the 80 scenario simulations at the spatial location of interest within the coastal model domain, and 3) historical distributions of offshore wave conditions to represent variability within each bin. The latter was required to compute statistical confidence limits on the constructed time-series.

For each climatological bin, normal distributions for wave height, period, and direction were computed using the mean and standard deviation of all data contained in the bin. At a specific time step within the desired time-series period, we found the probability, $p$, that a particular observation of significant wave height $\left(H_{s}\right)$ and mean wave direction $\left(\theta_{m}\right)$ corresponds to each climatological bin. The combined probability for each bin (denoted by the $j$ index), was defined as:

$p_{j}\left(H_{s}\right.$ and $\left.\theta_{m}\right)=p_{j}\left(H_{s}\right) * p_{j}\left(\theta_{m}\right)$.

This method assumes that wave height and direction were independent such that a wave of any size could come from any direction. The impact of this assumption is explored in Section 4.3. While a given offshore value of $H_{s}$ and $\theta$ can only fall within one bin, assigning a normal distribution to the observations in each climatological bin statistically accounts for times when the offshore wave conditions lie on a bin boundary and therefore would be expected to be best represented by some combination of the two scenarios on either side. In addition, this approach minimizes the error that would result if a small observational error would result in offshore wave conditions being falsely identified in one bin vs. an adjacent one.

For each point in time, the constructed wave parameters $\left(H_{s, r}, T_{s, r}, \theta_{s, r}\right)$ at a location anywhere within the model domain are then determined by:

$H_{s, r}=\sum_{j=1}^{80} p_{j}\left(H_{s}\right.$ and $\left.\theta_{m}\right) H_{s, m_{j}}$
$T_{s, r}=\sum_{j=1}^{80} p_{j}\left(H_{s}\right.$ and $\left.\theta_{m}\right) T_{s, m_{j}}$

$\theta_{s, r}=\sum_{j=1}^{80} p_{j}\left(H_{s}\right.$ and $\left.\theta_{m}\right) \theta_{s, m_{j}}$

where $H_{s,} m_{j}, T_{s}, m_{j}$, and $\theta_{s}, m_{j}$ are the modeled wave height, peak period, and mean direction, respectively, for each of the 80 model scenarios at the specified location inside the model domain.

The $90 \%$ confidence interval, used to define uncertainty in the constructed time-series, was computed by integrating $p_{j}\left(H_{s}\right.$ and $\left.\theta_{m}\right)$. While this analysis was focused on reconstructing historical timeseries, the same procedure could be used given wave projections or from specific weather scenarios.

\section{Results}

Time-series constructions were performed at two locations in the northern Gulf of Mexico and compared to observations of wave height, peak period, and mean or peak wave direction (depending on data availability). The constructed time-series are also compared to archived WAVEWATCH-III ${ }^{\circledR}$ reanalysis forecasts. A series of statistics are reported for each comparison including the bias, slope, and correlation coefficient $\left(r^{2}\right)$ determined by linear regression and the model skill (S) defined as in Garcez Faria et al. (1998) and Reniers et al. (2004),

$S=1-\sqrt{\frac{\frac{1}{N} \sum_{i=1}^{N}\left(\alpha_{m, i}-\alpha_{p, i}\right)^{2}}{\sqrt{\frac{1}{N} \sum_{i=1}^{N} \alpha_{m, i}^{2}}}}$

where $\alpha_{p, i}$ and $\alpha_{m, i}$ are the predicted and measured quantities, respectively. The index, $i$, spans from 1 to the total number of observations, $N$. A summary of the error statistics described in the following paragraphs is provided in Table 1 .

The longitudinally-separated wave observations are located in $30 \mathrm{~m}$ and $12 \mathrm{~m}$ water depths and are labeled in Fig. 1 as 42012 and ADCP, 
Table 1

Reconstruction performance

\begin{tabular}{llll}
\hline & & Buoy $42012(30 \mathrm{~m})$ & Santa Rosa ADCP $(12 \mathrm{~m})$ \\
\hline Bias & $H_{s}[\mathrm{~m}]$ & -0.02 & -0.01 \\
& $T_{p}[\mathrm{~s}]$ & -0.77 & -1.6 \\
& $\theta[\mathrm{deg}]$ & 7 & -9 \\
\hline \multirow{2}{*}{ RMSE } & $H_{s}[\mathrm{~m}]$ & 0.30 & 0.29 \\
& $T_{p}[\mathrm{~s}]$ & 1.6 & 3.0 \\
& $\theta[\mathrm{deg}]$ & 43 & 60 \\
\hline \multirow{2}{*}{ Slope } & $H_{s}$ & 0.76 & 0.72 \\
& $T_{p}$ & 0.54 & 0.26 \\
& $\theta$ & 0.65 & 0.30 \\
\hline$r^{2}$ & $H_{s}$ & 0.64 & 0.53 \\
& $T_{p}$ & 0.26 & 0.04 \\
& $\theta$ & 0.57 & 0.21 \\
\hline \multirow{2}{*}{ Skill } & $H_{s}$ & 0.67 & 0.58 \\
& $T_{p}$ & 0.71 & 0.53 \\
& $\theta$ & 0.76 & 0.65 \\
\hline
\end{tabular}

respectively. The first example uses wave observations from the National Data Buoy Center (NDBC) buoy 42012 from April 1, 2010 to December 31, 2012, which contains the time period used to define the climatological bins. A comparison of the time-series construction to all observations during this time period is shown along the left column of Fig. 4. The right-column highlights a portion of the constructed timeseries of significant wave height, peak period, and mean direction, along with the observations at the offshore buoy (NDBC 42040) which control construction of the inshore time-series.

The hourly significant wave height was predicted well with a bias of $2 \mathrm{~cm}$ and a statistically significant squared-correlation coefficient $\left(r^{2}\right)$ of 0.65 . Model skill $(S)$ is 0.67 with the greatest discrepancies for large wave heights. Separating the construction assessment to times before and after May 2012 does not significantly change the error statistics indicating that, in this case, the method was not sensitive to whether the construction time interval coincided with the time period of the underlying climatology used to define the model scenarios. Reconstruction skill for peak wave period is 0.71 and the $r^{2}$ value was low, but significant, at 0.26 . This skill was impacted, in part, by the discretization of the observed spectral peak. Reconstruction of mean wave direction is also skillful ( $S=0.76$ ) with some obvious scatter in the comparison (see Fig. 4). The general trends in wave direction, particularly rotation of the incident direction with the passing of storms, were predicted well.

To compare the probabilistic time-series construction performance against deterministic wave modeling techniques, the coastal wave model was run in time-variant mode using the same model parameterizations over three different continuous time periods ranging from 10 to 19 days. Wave and wind forcing was updated on three hour intervals with hourly water level updates including tides and forecasted subtidal components from the Hybrid Coordinate Ocean Model (HYCOM; 2012). Similar to the wave boundary conditions for the scenario simulations, archived WAVEWATCH-III ${ }^{\circledR}$ reanalysis output was used to prescribed the wave conditions at the offshore and lateral boundaries. The boundary spacing was kept the same as in the scenario simulations to ensure that any difference in the wave comparison is related to the technique rather than the boundary condition. The range in model skill for the deterministic simulations $(S=0.72-0.77,0.53-0.84$, and 0.51-0.63 for $H_{s}, T_{p}$, and $D_{m}$, respectively) at this site (bottom two panel rows of Fig. 5) was not significantly better than that for the probabilistic time-series construction method (compare to Table 1).

Lastly, the skill values from the probabilistic construction method were similar to those from the operational WAVEWATCH-III ${ }^{\circ}$ reanalysis forecasts for the same time period. Fig. 6 compares the performance
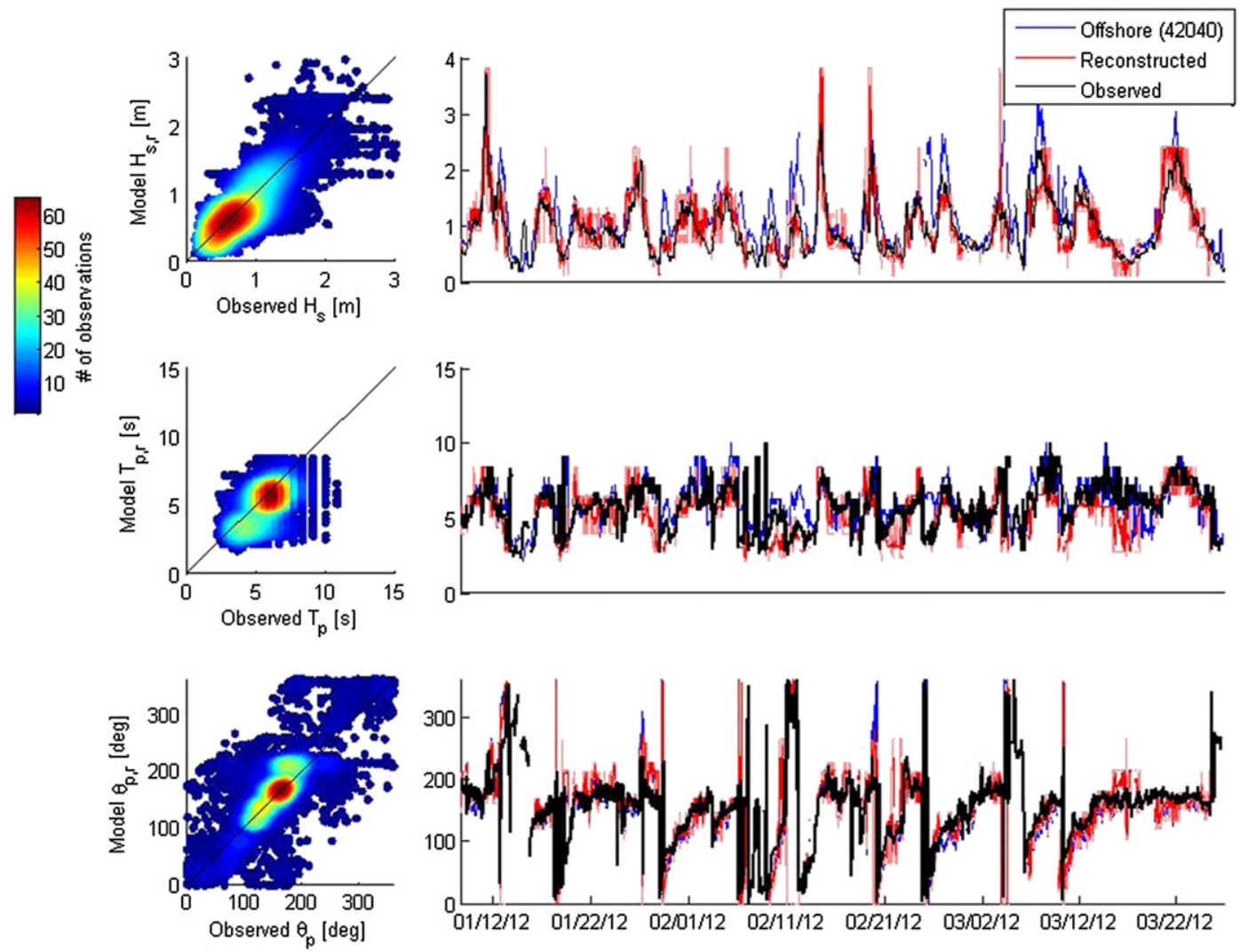



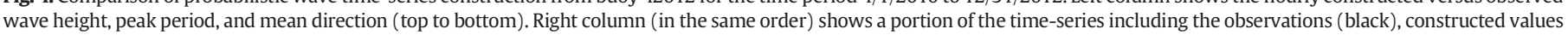
(red with $90 \%$ confidence interval shaded in pink), and measurement at offshore 42040 buoy (blue). 

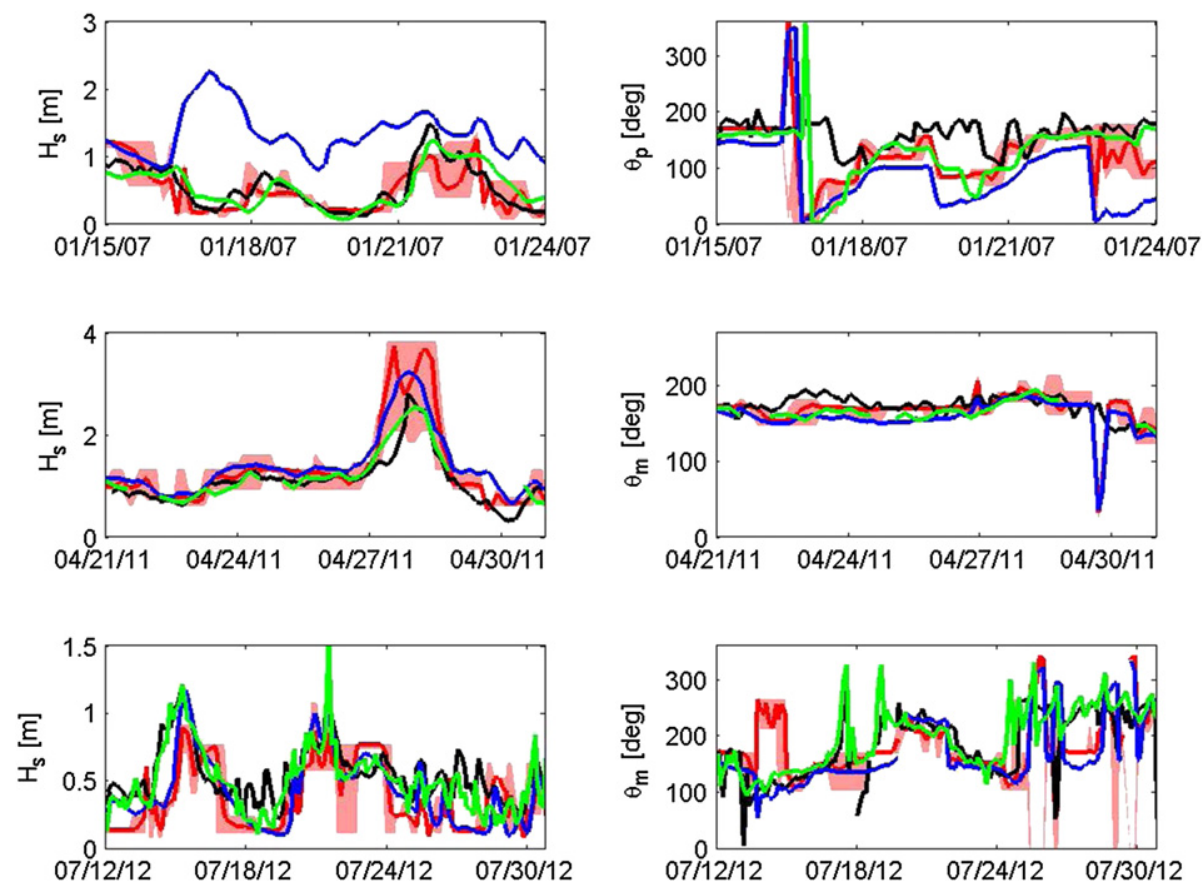

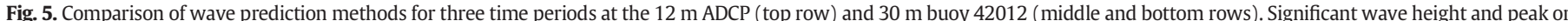

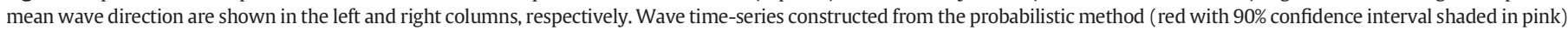

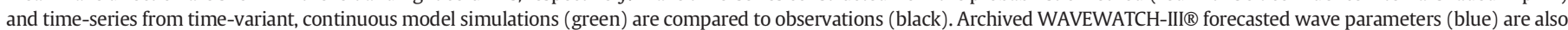
shown.

from the entire time period for wave height, period, and direction. The trends between the two methods were similar with a bit more scatter in the probabilistic construction, particularly at large wave heights. The results indicate that the probabilistic method has skill similar to
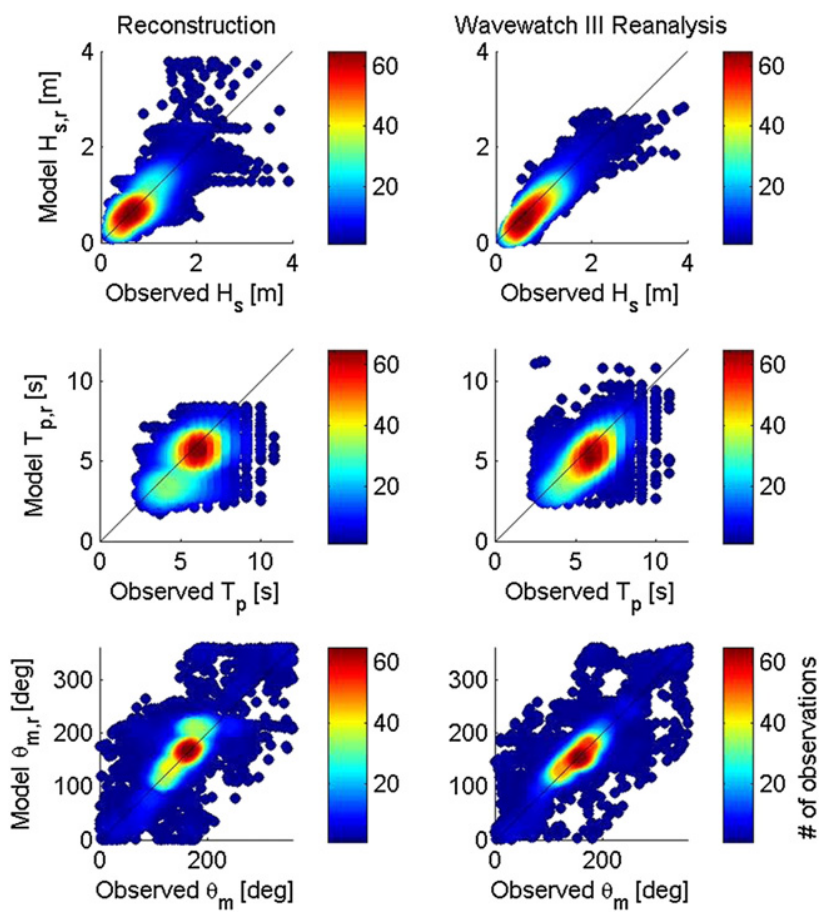

Fig. 6. Comparison of wave time-series constructed using the probabilistic method and observations at NDBC buoy 42012 for the time period 4/1/2010 to 8/1/2012 (left column). From top to bottom are comparisons of significant wave height, peak wave period, and mean wave direction. In the same order, a comparison of the WAVEWATCH-III® reanalysis dataset and the observations for the same period are shown in the right column. both deterministic model runs of similar spatial resolution, as well as to coarser resolution operational model output.

The second test location is located offshore of Santa Rosa Island, FL where an ADCP was deployed in approximately $12 \mathrm{~m}$ water depth from 2006 to 2007 (personal communication Todd Holland, Naval Research Laboratory, 2013). This example assesses the probabilistic time-series construction method 1 ) in shallower water depth which strongly impacts wave transformation and 2) during a time period outside the span used for the climatological assessment and original definition of model scenarios. Reconstruction of a portion of the 20-month wave time-series is shown in Fig. 7; right column.

A significant wave transformation between the offshore buoy 42040 and this site is apparent. While much of the temporal variation in wave height at this site corresponded to variations observed at the offshore buoy, there were times, particularly when wind/waves approached the offshore buoy from northern angles, when the offshore buoy measured large waves while the waves at the 12-m observational site were significantly smaller. This difference occurs because winds blowing from the north do not have enough fetch between the coastline and shallower locations to generate large waves. The probabilistic time-series construction method captured these phenomena, which would not be captured using a wave transformation method reliant on constant coefficients to transform offshore waves to an inshore location (see Fig. 7; around November 20, 2006).

The impact of refraction between the offshore buoy and the nearshore location can be seen in the shifting of peak wave direction toward more shore-normal (southerly) directions (Fig. 7; bottom right). This indicates that in addition to wave shoaling processes, modeled scenarios are sufficient to describe refraction patterns in the domain associated with a wide range of wave conditions. The model skill $(S=0.63,0.54$, and 0.65 for $H_{s}, T_{p}$, and $\theta_{p}$, respectively) is slightly lower than that of the $30-\mathrm{m}$ site, but still indicates the ability of this method to provide suitable nearshore boundary conditions, especially in locations where operational models may provide poor resolution of the sloping bathymetry near the coast. The reduction in skill for predicting wave direction may be affected by the comparison of peak versus mean spectral values 

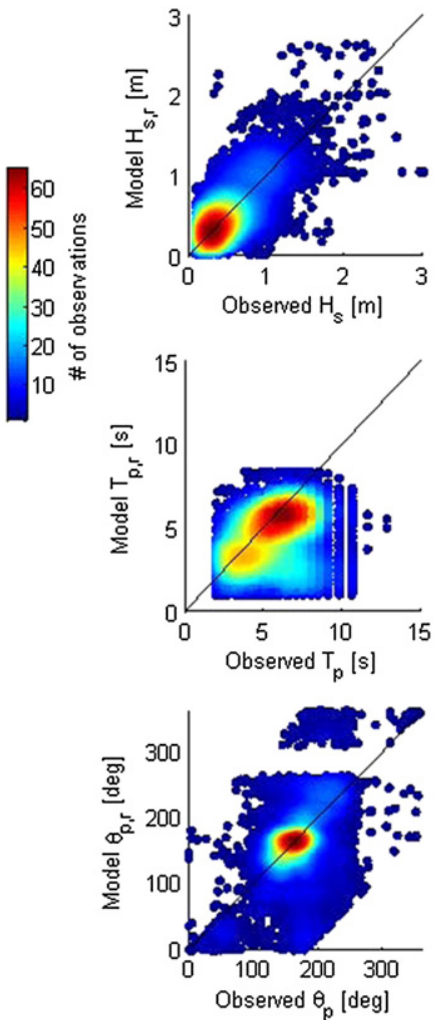
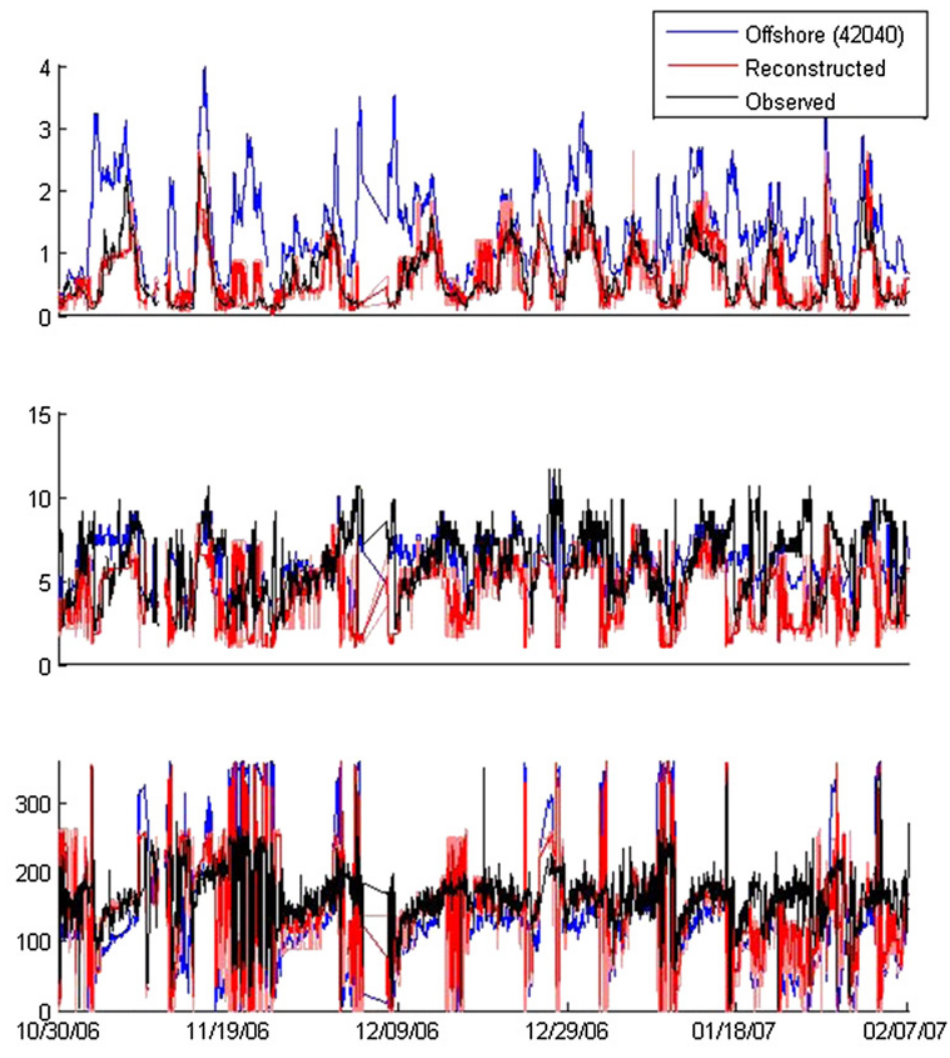

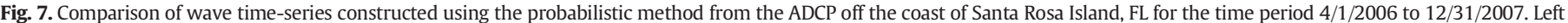

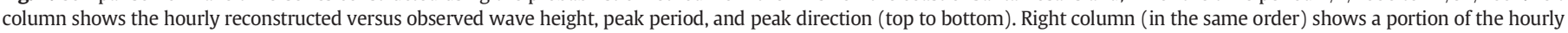
time-series from the observations (black), probabilistic construction (red with $90 \%$ confidence interval shaded in pink), and offshore buoy 42040 (blue).

due to data availability. Similar to the previous location, skill values for the probabilistic and time-varying deterministic simulations were not only comparable (Fig. 5; top panel row) but also much better than the operational forecast for significant wave height. Overall, the reduced skill in wave variables can also likely be attributed to the shallower water depth and temporal bathymetric variations not captured by the model.

\section{Discussion}

The method developed here provides the ability to construct skillful wave time-series and simultaneously provide confidence intervals on the wave predictions. Below we discuss the probabilistic time-series construction errors and confidence intervals and present sensitivity testing, particularly to the number of climatological bins and the number of model scenarios used to represent each bin. The impact of assuming independence between wave parameters is also discussed, followed by some limitations and potential future applications of the technique.

\subsection{Prediction uncertainty}

Observed wave height, peak period, and mean/peak direction at the shallow-water ADCP were contained within the $90 \%$ confidence limit calculated from the probabilistic time-series construction method 76 , 68 , and $77 \%$ of the time, respectively. For buoy 42012 , the data fell within the $90 \%$ confidence regions 78,68 , and $82 \%$ of the time. Thus, the uncertainty estimates are $8-22 \%$ too narrow and the construction is somewhat over-confident. Furthermore, the confidence regions vary in time, but did not show any clear trend that prediction error (i.e., mismatch between the best estimate from the probabilistic construction and the observations) increased as the confidence interval increased, suggesting that constant confidence intervals may suffice. The prediction errors at both sites did not significantly correlate with either the incident wave height or the direction at the offshore buoy. As mentioned previously, the uncertainty computed here is based on statistical distributions of the historical wave climate and does not include uncertainties due to bathymetric errors, model parameterizations, etc. While additional sources of uncertainty can be included in future developments, this method may offer improvement over other "look-up table" wave transformation methods (e.g., O'Reilly and Guza (1993)), in that we do not need to assume alongshore homogeneity, it is based on a climatological assessment of observed conditions, and at least some uncertainty in the prediction can be quantified.

We investigated the possibility of increased error when the offshore wave spectrum was bi-modal resulting in a mean direction which might poorly characterize some or all wave energy present. During these times there may be a significant amount of energy propagating from a particular direction that is not included by performing a time-series construction based on significant wave height and mean direction only. The wave time-series construction error was compared to a proxy for the presence of bi-modal spectra defined as the ratio of the mean and peak period observed offshore, with a value far from one indicating the presence of multiple peaks in the wave spectra. There was no significant correlation indicating that it is not a limiting factor in this area, but the impact and sensitivity may be greater at other coastal locations.

\subsection{Sensitivity to climatological binning}

We analyzed the sensitivity of the probabilistic time-series construction skill to the number of bins used to represent the climatological conditions and the number of model scenarios used to represent each 
climatological bin. Given the orientation of the coastline, one possible reduction in complexity was to use directional bins spanning only 90 to 270 , resulting in only 40 scenarios. Using this approach, the wave height construction at both inshore locations substantially overestimated the wave height during northern wave events. A summary of the skill for the probabilistic method as a function of the number of model scenarios used is shown in Fig. 8.

At the 12-m ADCP location, there was a noticeable improvement in predicting significant wave height when increasing the directional space to consider the full circle rather than only the southern 180 degree sector (40 versus 80 scenarios). Surprisingly, at the same site, the peak period and peak direction had slightly better skill under the 40 scenario, southern 180 degree case (e.g., Fig. 8; left column). With only using information at the offshore buoy to drive the probabilistic time-series construction, this most inshore location (12 m water depth) needed model scenarios showing that, during northern wave events when waves were large at the offshore buoy, they often remain small near the coast. Without these scenarios included, the wave height construction during these events significantly overestimates the wave height. However, when considering peak period and direction, it appears that the construction method benefits from having fewer possibilities and is best constrained when waves are only allowed to approach from south, southeast, and southwest directions which is consistent with the physical processes of waves propagating toward shore. At the $30 \mathrm{~m}$ buoy 42012 location, the predictive skill of peak period and mean direction is unaffected by including northern wave directions while significant wave height shows a slight increase in skill.

We also assessed the impact of using double or triple the number of scenario times when computing the probabilistic time-series construction. Rather than identifying only $1 \mathrm{~h}$ to represent each of the 80 bins, we identified three specific times when the offshore conditions represented the targeted (average) climatological bin values. Including more scenarios to represent each bin (e.g., maintaining 80 climatological bins but increasing from 80 to 160 or 240 model scenarios) did not, however, enhance the predictive skill for any of the variables
(Fig. 8). While the same mean condition for the matching was used, different spatial patterns over the large domain existed. Finally, a probabilistic construction based on only the most likely scenario value for each hour (the bin in which the observed offshore condition fell), rather than the weighted sum of probability from all scenarios, showed some degradation in skill. It is possible that further discretizing the largest climatological wave height bin $(2<H<5 \mathrm{~m})$ would provide a better wave construction for larger wave heights. With the current climatological binning at buoy 42040 , both winter cold fronts and summer tropical storms passing through this location are sometimes contained in the same wave height-direction bin, despite their different spatial and propagation characteristics.

\subsection{Comparison to Bayesian approach}

When performing the multi-variate scenario matching, it is possible to extend the technique to include wave period and wind speed/direction in computing the probability that a certain time falls within one of the available scenarios. Initial attempts revealed a degradation in reconstructive skill by imposing additional constraints with variables not resolved with the climatological binning, potentially in part a result of the variable dependency (e.g., wind speed and wave height). An alternate, machine-learning probabilistic method to predict wave conditions at alternate locations that does not assume independence when constructing distributions of wave height and wave direction (as the probabilistic time-series construction introduced here) is to use Bayes' rule (Plant and Holland, 2011a). The Bayesian method can take into account full distributions of all relevant variables (winds, wave period, bathymetry, etc.), but a training set is required at any location of interest. Unlike the probabilistic time-series construction technique described here, the Bayesian approach is too computationally expensive to run over a large spatial domain using model scenarios. Fig. 9 compares the performance of the construction method described here with the performance of a Bayesian-derived time-series (see Plant and Holland, 2011a,b) at buoy 42012. Results indicate that except at large
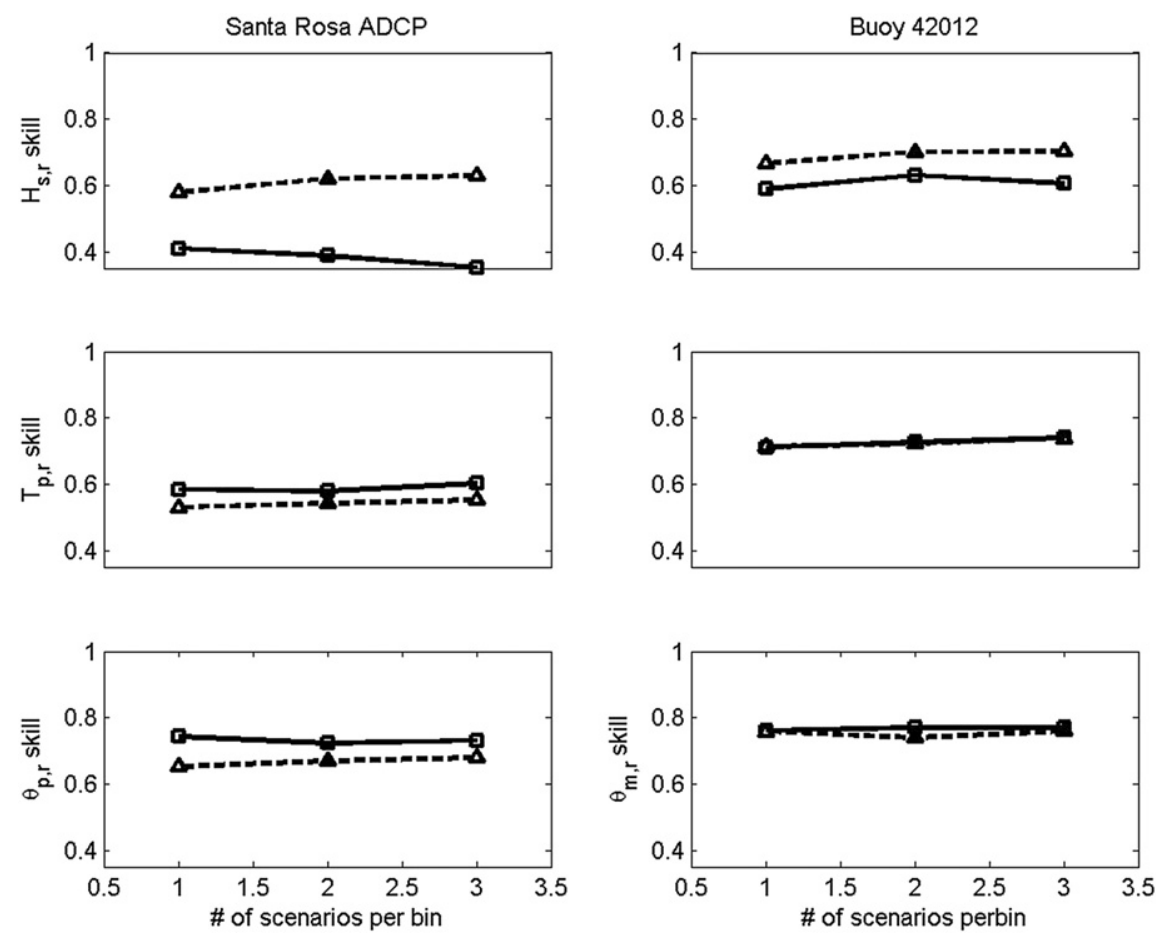

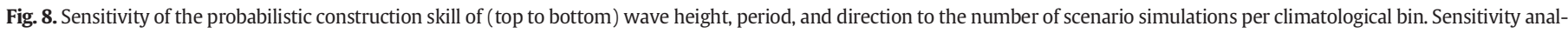

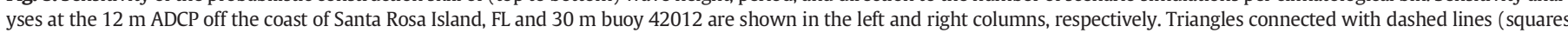
connected with solid lines) represent the model skill using scenarios with wave directions spanning 0-360 (90-270) degrees. 

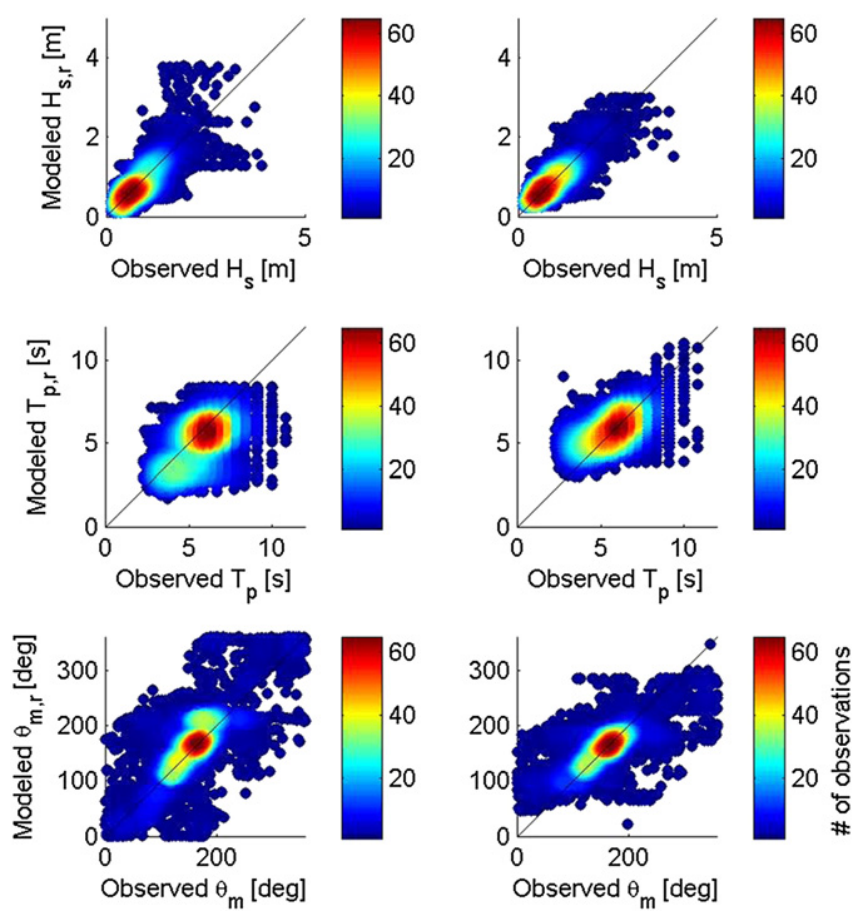

Fig. 9. Comparison of probabilistic wave time-series construction (left column) and a full Bayesian-derived time-series (right column) at NDBC buoy 42012 for the time period 4/1/ 2010 to 12/31/2012 (left column). From top to bottom are comparisons of significant wave height, peak wave period, and mean wave direction. Colors indicate the density of observations.

wave heights, this simplified probabilistic construction method performs as well as the full Bayesian approach with somewhat increased performance in predicted wave direction.

\subsection{Potential applications and limitations}

The technique developed here is strictly valid for conditions where the water depths are effectively constant, such as outside of the surf zone. For situations where the bathymetry is changing, one could add bathymetric scenarios in addition to the scenarios derived from a climatological assessment. This has been demonstrated to be effective in applications with very limited spatial extent (Plant and Holland, 2011b). Furthermore, the limitation of accurate bathymetry is inherent in conventional modeling systems as well due to limited availability of up-to-date bathymetric data. Nonetheless, the present approach can be used to supply boundary conditions for surf zone models.

While the results presented here show that a wave time-series can be constructed for a time period not included in the assessment of the wave climatology (Section 3; 12-m location), the time-series construction skill may degrade if the climatology is built on anomalous time periods or the method is used to represent extreme conditions outside of the variability captured by the database of scenarios.

Applications of this technique can be expanded to include climate change studies, storm scenarios, operational activities where uncertainty is required, and design situations. For example, the method has been previously applied to the application of assessing the mobility of sand and oil agglomerates found along the coast following the Deepwater Horizon oil spill (Plant et al., 2013). Time series of nearshore wave conditions close to coast were constructed and used to determine weighted probabilities of formation, mobility, and transport in different regions of the model domain (Dalyander et al., 2014). The same could be applied to future oil spills. While that study extended this work to reconstruct time-series of alongshore current magnitude/direction in the surf zone, additional research is required for validation and improvement of the technique. In addition, the impact of potentially increasing offshore wave heights or extreme events (e.g., Ruggiero et al., 2010) to the coastal region can be assessed.

\section{Conclusions}

We have developed an efficient probabilistic time-series construction method to model time-series of wave characteristics, particularly height, period, and direction, at different spatial locations given offshore point observations and a database of deterministic numerical wave model scenario simulations. These modeled time-series are computed without additional direct numerical simulation and have skill similar to that of archived operational simulations at higher spatial resolution. Model skill is also comparable to a machine-learning, Bayesian approach that cannot create time-series at locations without training data and is computationally too expensive to use to generate timeseries at many locations over large spatial domains. The predictions presented here require an archived set of numerical simulations based on a buoy-derived wave climatology, basin-scale wave model output, and offshore wave conditions during the time period of interest either from a buoy, offshore wave model, or other estimate of future or past conditions. Sensitivity analysis indicates that construction of wave time-series based on adding more than the 80 scenarios used here provides negligible improvement. The potential applications of this method are widespread among engineering and scientific disciplines. In addition to its use in hindcasting wave parameters, analysis of future scenarios can be modeled anywhere in the domain using the scenario simulations.

\section{Acknowledgments}

Any use of trade, firm, or product names is for descriptive purposes only and does not imply endorsement by the U.S. Government. This work was funded by the United States Coast Guard and the United States Geological Survey. We thank Todd Holland for providing the Santa Rosa ADCP data used in this work and Li Erikson for her review of the initial manuscript. The two anonymous reviewers also provided helpful and constructive feedback.

\section{References}

Battjes, J.A., Janssen, J.P.F.M., 1978. Energy loss and set-up due to breaking random waves. Proceedings, 16th International Conference on Coastal Engr., ASCE, pp. 68-81.

Booij, N., Ris, R., Holthuijsen, L., 1999. A third-generation wave model for coastal regions: 1. Model description and validation. J. Geophys. Res. 104, 7649-7666.

Camus, P., Mendez, F.J., Medina, R., Cofiño, A.S., 2011. Analysis of clustering and selection algorithms for the study of multivariate wave climate. Coast. Eng. 58, 453-462.

Dalyander, P.S., Long, J.W., Plant, N.G., Thompson, D.M., 2014. Assessing mobility and redistribution patterns of sand and oil agglomerates in the surf zone. Mar. Pollut. Bull. 80, 200-209.

Defne, Z., Haas, K.A., Fritz, H.M., 2009. Wave power potential along the Atlantic coast of the southeastern USA. Renew. Energy 34, 2197-2205 (URL: http://www. sciencedirect.com/science/article/pii/S0960148109000871, http://dx.doi.org/10. 1016/j.renene.2009.02.019)

Garcez Faria, A.F., Thornton, E.B., Stanton, T.P., Soares, C.V., Lippmann, T.C., 1998. Vertical profiles of longshore currents and related bed shear stress and bottom roughness. J. Geophys. Res. 103, 3217-3232.

Hasselmann, K., Barnett, T., Bouws, E., Carlson, H., Cartwright, D., Enke, K., Ewing, J., Gienapp, H., Hasselmann, D., Kruseman, P., et al., 1973. Measurements of windwave growth and swell decay during the Joint North Sea Wave Project (JONSWAP).

Komen, G., Hasselmann, S., Hasselmann, K., 1984. On the existence of a fully developed wind-sea spectrum. J. Phys. Oceanogr. 14, 1271-1285.

Londhe, S., 2008. Soft computing approach for real-time estimation of missing wave heights. Ocean Eng. 35, 1080-1089.

Londhe, S., Panchang, V., 2007. Correlation of wave data from buoy networks. Estuar. Coast. Shelf Sci. 74, 481-492.

O'Reilly, W., Guza, R.T., 1993. A comparison of two spectral wave models in the Southern California Bight. Coast. Eng. 19, 263-282.

Plant, N.G., Holland, K.T., 2011a. Prediction and assimilation of surf-zone processes using a Bayesian network: part I: forward models. Coast. Eng. 58, 119-130.

Plant, N.G., Holland, K.T., 2011b. Prediction and assimilation of surf-zone processes using a Bayesian network: part II: inverse models. Coast. Eng. 58, 256-266.

Plant, N.G., Long, J.W., Dalyander, P.S., Thompson, D.M., 2013. Hydrodynamic and sediment transport model application for OSAT3 guidance. U.S. Geological Survey Open-File Report 2012-1234. U. S. Geological Survey. 
Reikard, G., 2009. Forecasting ocean wave energy: tests of time-series models. Ocean Eng. $36,348-356$

Reniers, A.J.H.M., Thornton, E.B., Stanton, T.P., Roelvink, J.A., 2004. Vertical flow structure during Sandy Duck: observations and modeling. Coast. Eng. 51, 237-260. http://dx. doi.org/10.1016/j.coastaleng.2004.02.001.

Roelvink, D., Reniers, A., van Dongeren, A., van Thiel de Vries, J., McCall, R., Lescinski, J., 2009. Modelling storm impacts on beaches, dunes and barrier islands. Coast. Eng. $56,1133-1152$.

Ruggiero, P., Komar, P.D., Allan, J.C., 2010. Increasing wave heights and extreme value projections: the wave climate of the U.S. Pacific Northwest. Coast. Eng. 57, 539-552 (URL: http://www.sciencedirect.com/science/article/pii/S0378383909002142, http://dx.doi. org/10.1016/j.coastaleng.2009.12.005).
Stockdon, H.F., Holman, R.A., Howd, P.A., Sallenger, A.H., 2006. Empirical parameterization of setup, swash, and runup. Coast. Eng. 53, 573-588.

Stockdon, H., Doran, K., Thompson, D., Sopkin, K., Plant, N., Sallenger, A., 2012. Nationa assessment of hurricane-induced coastal erosion hazards - Gulf of Mexico. Technical Report 2012-1084. U.S. Geological Survey Open-File Report.

Tolman, H.L., 2008. A mosaic approach to wind wave modeling. Ocean Model. 25, 35-47 (URL: http://www.sciencedirect.com/science/article/pii/S1463500308000863, http:// dx.doi.org/10.1016/j.ocemod.2008.06.005.)

Yates, M., Guza, R., OReilly, W., 2009. Equilibrium shoreline response: observations and modeling. J. Geophys. Res. 114, C09014. 